* Corresponding author Phone + 21674274409

E-mail address:mohamed.mseddi@fss.rnu.tn (M. Mseddi) or zied.driss@enis.rnu.tn (Z.Driss)

Article information

Article history: AMS-Volume15-No.2-00110-1 Received 05 April 2011

Accepted 18 May 201

Available online 26 August

\section{Computer Simulations of the Static State of the Turbocharger Turbine}

\author{
M. Mseddi ${ }^{\text {b* }}$, Z. Driss ${ }^{\text {a }}$, M. Baccar ${ }^{\text {b }}$, M.S. Abid ${ }^{\text {a }}$ \\ a Unit of Computational Fluid Dynamics \& Transfer Phenomena (CFDTP), National Engineering School of Sfax, B.P. 1173 Sokra 3038, Sfax, \\ Tunisia \\ ${ }^{b}$ Laboratory of Electro-Mechanic Systems (LASEM), National Engineering School of Sfax, B.P. 1173 Sokra 3038, Sfax, Tunisia
}

\section{KEY WORDS}

CFD, Simulation, Turbulent flow, Turbocharger, Turbine, Static state

\begin{abstract}
In the present work, a numerical study of a turbulent flow through a turbocharger turbine in a static state is presented. Solutions of the time averaged Navier-Stokes in conjunction with the standard $\mathrm{k}-\varepsilon$ turbulence model were developed using a control volume discretization method. The resolution was found by the commercial code Fluent 5.6. Geometry and the meshing were built by the software Gambit 6.1. This study is expected to provide a finer knowledge of structures of the flow such as velocity, pressure, turbulent kinetic energy, dissipation rate of the turbulent kinetic energy and viscosity. The proposed model has been tested on a turbocharger turbine of type Garrett TA03 automotive engines. The numerical results have been compared with ones found by other experimental results.
\end{abstract}

\section{Introduction}

Several studies have investigated turbocharger turbines. Examples of these are Fevre (Fevre, 1990), Kesgin (Kesgin, 2005), Rakopoulos et al. (Rakopoulos et al., 2004), Katrasnik (Katrasnik, 2006), Sarvi et al. (Sarvi et al., 2008), Lee et al. (Lee et al., 2002), Serrano et al. (Serrano et al., 2008), Podevin (Podevin, 1983), Friberg et al. (Friberg et al., 1988), Frelin and Podevin (Frelin et al., 1992), Schorn et al. (Schorn et al., 1987), Mseddi et al. (Mseddi et al., 2005; Mseddi, 1990) and Driss et al. (Driss et al., 2003; Driss, 2004). Kesgin (Kesgin, 2005) presented the results of investigations to design the inlet and exhaust systems of a stationary natural gas engine family. To do this, a computational model is verified in which zero dimensional phenomena within the cylinder and one dimensional phenomena in the engine inlet and exhaust systems are used. Using this engine model, the effects of the parameters of the inlet and exhaust systems on the engine performance are obtained. Rakopoulos et al. (Rakopoulos et al., 2004) developed a computer analysis for studying the energy and availability performance of a turbocharged diesel engine, operating under transient load conditions. The model incorporates many novel features for the simulation of transient operation, such as detailed analysis of mechanical friction, separate consideration for the processes of each cylinder during a cycle and mathematical modeling of the fuel pump. This model has been validated within experimental data taken from a turbocharged diesel engine operated under transient conditions. The availability terms for the diesel engine and its subsystems are analyzed. Katrasnik (Katrasnik, 2006) presented an innovative algorithm for the simulation of a turbocharger gas turbine and compressor based on extended equations of the method of character- 
istics. This algorithm enables consideration of variable inlet and outlet pressure as well as consideration of variable gas properties and concentrations. Sarvi et al. (Sarvi et al., 2005) measured gaseous emissions (NOx, $\mathrm{CO}, \mathrm{HC})$ according to the international maritime organisation technical code. They determined optically smoke emissions and they measured particulate matter using a gravimetric impactor for five size fractions. Results show that the exhaust emission was also highly dependent on the engine turbocharger system, especially the by-pass control, but was not affected by waste gate control. Lee et al. (Lee et al., 2002) investigated the improvement of transient characteristics of a turbocharged diesel engine under the conditions of low speed and fast acceleration with the load. The experiment for improving the low speed torque and acceleration performance is performed by means of injecting air into the intake manifold during the period of low speed and application of a fast acceleration. At low speed range of the engine, the effect of air injection shows the improvement of the pressure distribution of turbocharger and combustion pressure during the period of gas exchange pressure. Serrano et al. (Serrano et al., 2008) presented a model of fixed and variable geometry turbines. The aim of this model is to provide an efficient boundary condition to model turbocharged internal combustion engines with zero and one dimensional gas dynamic codes. The model is based from its conception on the measured characteristics of the turbine. Nevertheless, it is capable to extrapolate operating conditions that differ from those included in the turbine maps, since the engines usually work within these zones. The presented model has been implemented in a one dimensional gas dynamic code and has been used to calculate unsteady operating conditions for several turbines. The results obtained have been compared with success against pressure-time histories measured upstream and downstream of the turbine during engine operation. Also, turbines with variable geometry were appeared in many anterior works. In fact, Mazaud (Mazaud, 1990) tested the process of combined overfeeding whose results proved to be disappointed in cases where a release through a wastegate is required. In the same way, Okazaki (Okazaki, 1992) tested a turbine with mobile channels distributed on the periphery of the dispenser. He showed that the outputs are similar to those obtained from the configuration of reference and sweep a larger mass flow envelope. In the brutal increase of pressure and flow, the turbocharger turbine functions a short moment under conditions similar to those of the measurements taken in a static state by Fevre (Fevre, 1990). These results are very interesting to understand what occurs in the wheel in the case of a very significant loss of adaptability. In addition, Mseddi et al. (Mseddi et al., 1992; Mseddi, 2004) demonstrated in the analytical study of a radial flow turbine, that the constant on which the calculation of the detente rate can be determinate from the local results of a numerical study of the real flow in a radial turbine. Then, the study of the flow through the turbocharger turbine remains interesting in a static state. In these conditions, the wheel is blocked. Thus, we can see the influence of fixed paddle on the flow. In addition, while placing the wheel in the volute but without ability to turn, we undoubtedly modified the flow in a significant way. But, it's noted that the real conditions of power supply of the turbine are very variable. Furthermore, the knowledge of the flow in the rotor is interesting to make measurements between the blades ahead in the wheel. But measurements are not realizable without involving significant modifications on the volute for the passage of the beams. In addition, there is partial covering of the blades, which makes impossible all optical measurements. Then, solutions of the averaged Navier-Stokes in conjunction with the standard $\mathrm{k}-\varepsilon$ turbulence model were developed using a control volume discretization method. The resolution was found by the commercial code Fluent 5.6. Geometry and the meshing were built by the software Gambit 6.1. This study is expected to provide a finer knowledge of structures of the flow such as velocity, static pressure, turbulent kinetic energy $\mathrm{k}$, dissipation rate of the turbulent kinetic energy $\varepsilon$ and effective viscosity $v_{\mathrm{e}}$. A comparison between results obtained by the computational fluid dynamics (CFD) code and those obtained by Podevin application is presented (Podevin, 1983).

\section{Numerical model \\ 2.1 Navier-Stokes equations}

The equations governing the turbulent flow are the classical Navier-Stokes. The instantaneous turbulent field is decomposed classically in an average and a fluctuant component. The appropriate equations are expressed in the general conservation form which can be written as follows:

$$
\frac{\partial \rho}{\partial \mathrm{t}}+\operatorname{div}(\rho \overrightarrow{\mathrm{V}})=0
$$




$$
\begin{aligned}
& \frac{\partial(\rho \overrightarrow{\mathrm{V}})}{\partial \mathrm{t}}+\operatorname{div}(\rho \overrightarrow{\mathrm{V}} \otimes \overrightarrow{\mathrm{V}}+\mathrm{P} \overline{\overline{\mathrm{I}}})= \\
& =\operatorname{div}(\bar{\tau}+\tau \mathrm{R}) \\
& \frac{\partial(\rho \mathrm{E})}{\partial \mathrm{t}}+\operatorname{div}(\rho \mathrm{E} \overrightarrow{\mathrm{V}}+\mathrm{P} \overrightarrow{\mathrm{V}})= \\
& =\operatorname{div}[(\tau+\tau)=\overline{\mathrm{R}}) \overrightarrow{\mathrm{V}}]
\end{aligned}
$$

$\overline{\bar{\tau}}=-\frac{2}{3} \mu(\operatorname{div} \overrightarrow{\mathrm{V}}) \overline{\overline{\mathrm{I}}}+2 \mu \overline{\overline{\mathrm{D}}}$

$\overline{\overline{\mathrm{D}}}=\frac{1}{2}\left[\nabla \overrightarrow{\mathrm{V}}+(\nabla \overrightarrow{\mathrm{V}})^{\mathrm{T}}\right]$

In these expressions $\rho, \mathrm{V}$ and $\mathrm{P}$ designate the average values of the mass volume, the velocity and the static pressure. The total average energy $\mathrm{E}$ is then the sum of the total energy of the average movement and the kinetic energy of the turbulent movement $\mathrm{k}$ :

$\mathrm{E}=\left(\mathrm{u}+\frac{1}{2} \mathrm{~V}^{2}\right)+\mathrm{k}$

For a perfect gas, the static pressure is given by the law of state:

$\mathrm{P}=\rho \mathrm{RT}=(\gamma-1) \rho \mathrm{u}$

The average stress tensor is presented by $\tau$ and has for expression: where: $\mu(u)$ coefficient of molecular viscosity, I unit tensor, $\mathrm{D}$ distortions tensor of the average movement.

In relation to the instantaneous Navier-Stokes equations, the average equations produced two supplementary terms: the kinetic energy $\mathrm{k}$ and the tensor of Reynolds $\tau_{\mathrm{R}}$.

\subsection{Turbulence model}

The turbulence model used is a $\mathrm{k}-\varepsilon$ type. This model is used in many papers. For example, Kreuz-Ihli et al. (Kreuz-Ihli et al., 2000) had used this model to study the flow in a radial turbine by the commercial code TASCflow3D. These results showed good satisfaction. Equations of the turbulent kinetic energy $k$ and the dissipation rate of the turbulent kinetic energy $\varepsilon$ can be written as follows:

$\frac{\partial \rho \mathrm{k}}{\partial \mathrm{t}}+\operatorname{div}[\rho \overrightarrow{\mathrm{V}} \mathrm{k}]=\operatorname{div}\left[\left(\mu+\frac{\mu_{\mathrm{t}}}{\sigma_{\mathrm{k}}}\right) \nabla \mathrm{k}\right]+\underset{\bar{\tau} \mathrm{R}: \nabla \overrightarrow{\mathrm{V}}}{=} \rho \varepsilon$

$\frac{\partial \rho \varepsilon}{\partial \mathrm{t}}+\operatorname{div}[\rho \overrightarrow{\mathrm{V}} \varepsilon]=\operatorname{div}\left[\left(\mu+\frac{\mu_{\mathrm{t}}}{\sigma_{\varepsilon}}\right) \nabla \varepsilon\right]+\mathrm{C}_{1} \frac{\varepsilon}{\mathrm{k}} \stackrel{=}{\tau} \mathrm{R}: \nabla \overrightarrow{\mathrm{V}}-\mathrm{C}_{2} \rho \frac{\varepsilon^{2}}{\mathrm{k}}$

$\mathrm{C} 1, \mathrm{C} 2, \mathrm{C} \mu, \sigma \mathrm{k}$ and $\sigma \varepsilon$ are the constants of the $\mathrm{k}-\varepsilon$ model.

The tensor of Reynolds $\tau_{\mathrm{R}}$ is formulated by:

$\bar{\tau} \overline{\mathrm{R}}=-\frac{2}{3} \mu_{t}(\operatorname{div} \overrightarrow{\mathrm{V}}) \overline{\overline{\mathrm{I}}}+2 \mu_{t} \overline{\overline{\mathrm{D}}}$

The coefficient of turbulent viscosity $\mu_{t}$ is function of the $\mathrm{k}$ and $\varepsilon$. This coefficient is given by:

$$
\mu_{t}=C_{\mu} \rho \frac{k^{2}}{\varepsilon}
$$

\subsection{Geometry System}

The proposed model has been tested on a turbocharger turbine automotive engines Garrett TA03 type. This turbine is provided with 11 blades. In this study, these blades are supposed flat (Fig. 1).

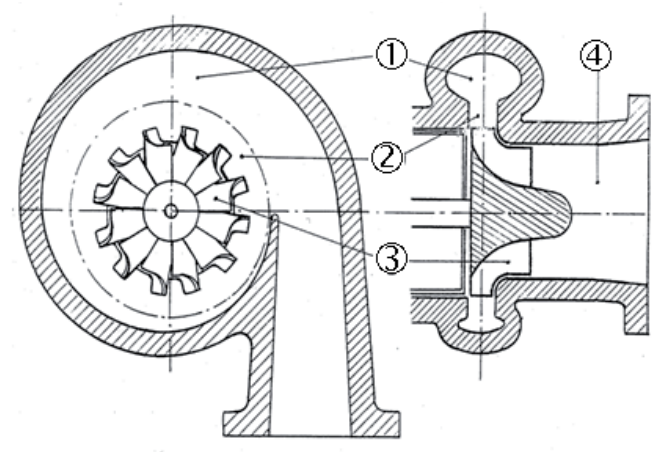

(1) Volute (2)Distributor (3)Wheel (4)Diffuser

Fig. 1: Geometrical configuration of the turbocharger turbine Garrett TA03.

\subsection{Boundary Conditions}

Figure 2 show the grid of the symmetry plane of the volute which contains the blades. 3D geometry of the turbocharger turbine, as well as the meshing of the do- 
main was built by the software Gambit. On the other hand, the mass flow in the inlet and the static pressure in the outlet were imposed. Air was supposed to be perfect and its properties were obtained from the kinetic theory of gases.
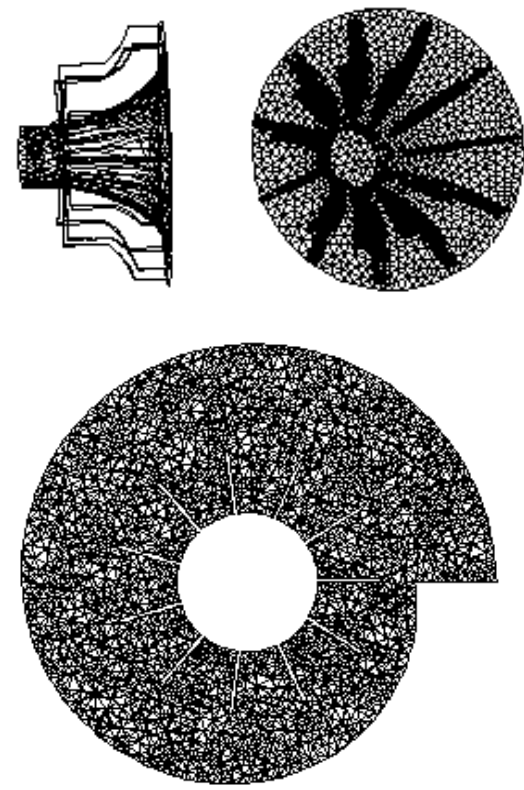

Fig. 2: Meshing of the turbocharger turbine.

\section{Numerical results}

The results are provided for a flow of $30 \mathrm{~g} . \mathrm{s}^{-1}$, a density of $1.23 \mathrm{~kg} . \mathrm{m}^{-3}$, a dynamic viscosity of $1.7910^{-5}$ $\mathrm{kg} \cdot \mathrm{m}^{-1} \cdot \mathrm{s}^{-1}$ and a total pressure at the volute inlet of $105532 \mathrm{~Pa}$. This study attempted to provide a better knowledge of the structures of the flow such as velocity, static pressure, turbulent kinetic energy k, dissipation rate of the turbulent kinetic energy $\varepsilon$ and effective viscosity $v_{\mathrm{e}}$ for a Reynolds number Re equal to 3.74 $10^{5}$.

\subsection{Flow characteristics in the wheel inlet}

In the wheel inlet defined by $\mathrm{r}=33.6 \mathrm{~mm}$ and for various azimuths equals to $\theta=90^{\circ}, \theta=180^{\circ}, \theta=360^{\circ}$ and the evolution of the various characteristics of the flow is presented. The evolution of the radial velocity is presented in Fig. 3. This figure shows that the curves are not symmetrical. Thus, for a given azimuth, the radial velocity which decreases gradually by the hub $(\mathrm{z}<0)$ towards the case, depends on the azimuth. In addition, near to the hub the radial velocity is more important while the azimuth get weaker. But, for the azimuth $360^{\circ}$, the radial velocity increased quickly passing from

\section{$1.5 \mathrm{~m} \cdot \mathrm{s}^{-1}$ to $15 \mathrm{~m} \cdot \mathrm{s}^{-1}$.}

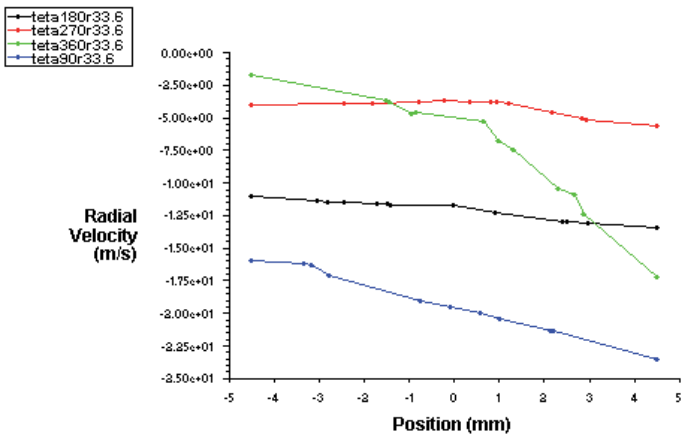

Fig. 3: Radial velocity for various azimuths.

Figure 4 shows the evolution of the tangential velocity for an azimuth ranging between $90^{\circ}$ and $270^{\circ}$. This compound is positive, depending little on the scale and is lower while the azimuth is larger. On the other hand, for the azimuth $360^{\circ}$, the tangential velocity is negative. Figure 5 shows the evolution of the axial velocity for various azimuths. Like to the radial velocity, this compound was found to be not symmetrical. However, the axial velocity values were very low. Thus, in the center $(\mathrm{z}=0)$, it was not equal to zero.

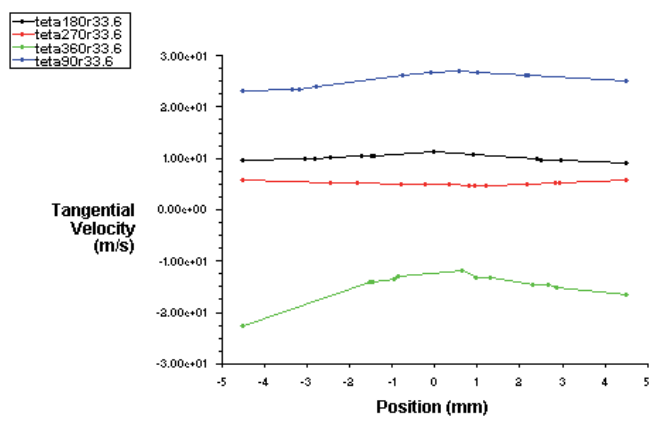

Fig. 4: Tangential velocity for various azimuths.

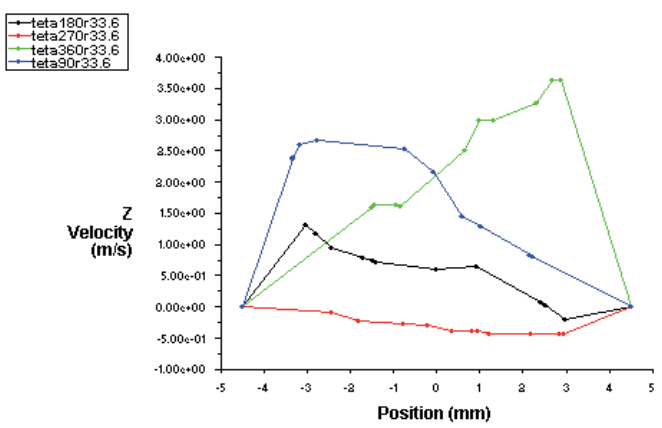

Fig. 5: Axial velocity for various azimuths. 
Figure 6 shows the evolution of the average velocity for various azimuths. At the wheel outlet, the profiles of the average velocity were not symmetrical. In the center, it was not completely constant and it was slightly more significant near the hub. While approaching the walls, the average velocity decreased until being equal to zero. The average velocity decreased for the azimuth $90^{\circ}$ to the azimuth $270^{\circ}$, and increased for the azimuth $270^{\circ}$ to the azimuth $360^{\circ}$.

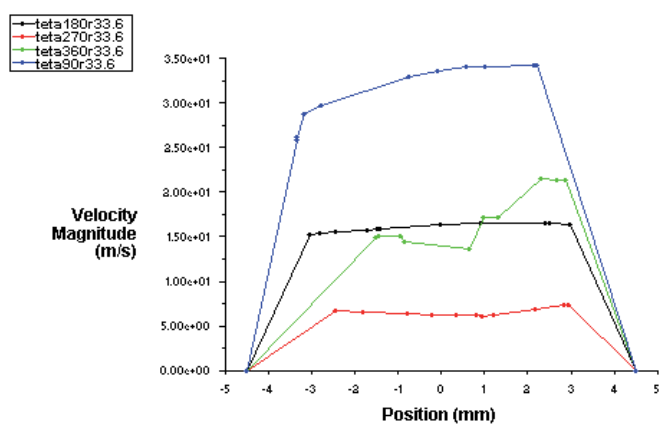

Fig. 6: Average Velocity for various azimuths.

Figure 7 shows the evolution of the turbulent kinetic energy $\mathrm{k}$. For the azimuth $360^{\circ}, \mathrm{k}$ was significant with a maximum value in the central zone of about $140 \mathrm{~m}^{2} \cdot \mathrm{s}^{-2}$.

In addition, the turbulent kinetic energy was relatively weak. However, $k$ decreased in the azimuth sector $90^{\circ}-270^{\circ}$ when the azimuth increased. Figure 8 shows the evolution of the dissipation rate of the turbulent kinetic energy $\varepsilon$ for various azimuths. As same as the turbulent kinetic energy $\mathrm{k}$, $\varepsilon$ was very large for the azimuth $360^{\circ}$. The highest value is located near the casing for about $1.810^{6} \mathrm{~m}^{2} . \mathrm{s}^{-3}$. In addition, $\varepsilon$ was weak.

Figure 9 shows the evolution of the effective viscosity ve for various azimuths. In the central zone, the values of $v_{\mathrm{e}}$ were the highest. Near the walls, it was low. In the same way, the maximum value was obtained for the azimuth equal to $\theta=180^{\circ}$.

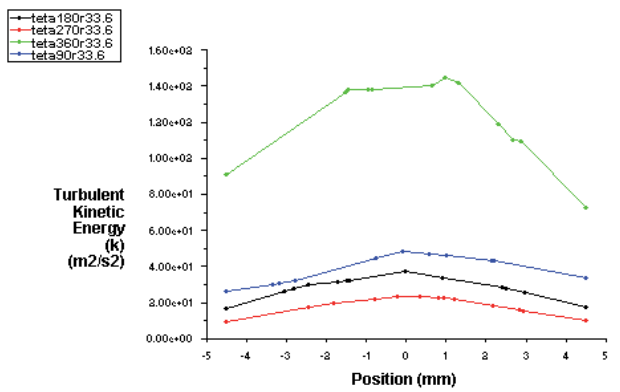

Fig. 7: Evolution of the turbulent kinetic energy k for various azimuths.

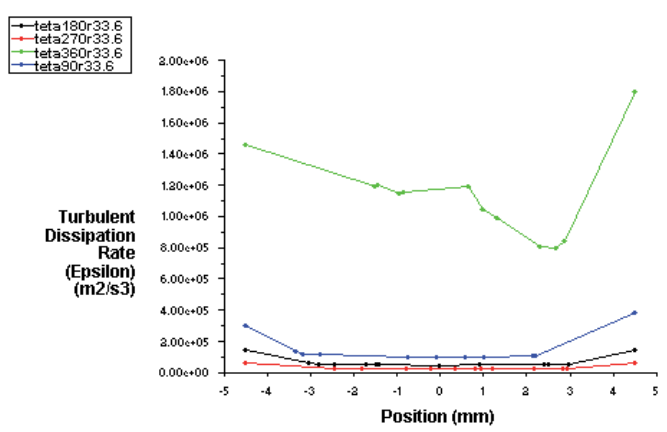

Fig. 8: Evolution of the dissipation rate of the turbulent kinetic energy $\varepsilon$ for various azimuths.

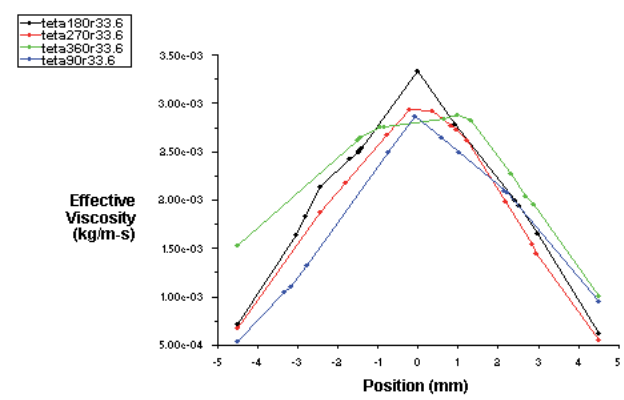

Fig. 9: Effective viscosity ve for various azimuths.

In the same azimuth sector $\left(180^{\circ}\right), \mathrm{k}$ and $v_{\mathrm{e}}$ reached their most significant values. Out of this sector, these values have dropped. The most significant values of the dissipation rate of the turbulent kinetic energy $\varepsilon$ were reached near the walls.

\subsection{Flow characteristics in the symmetry plane of the volute and the distributor}

In the symmetry plane from the volute and the distributor $(\mathrm{z}=0)$, the evolution of the various characteristics of the flow is presented. Figure 10 shows the distribution of the radial velocity. In the azimuth sector $0^{\circ}-180^{\circ}$ and between the blades, the flow was centripetal. The maximal value was obtained between the blades 1 and 2. In addition, the flow was centrifugal in the upstream of the blade 1 , in the downstream of the blades 2, 3 and 4, and between the blades 10 and 11 .

Figure 11 shows the distribution of the tangential velocity. The maximal value of this compound was obtained in the volute inlet and it reached $53 \mathrm{~m} \cdot \mathrm{s}^{-1}$. In the wheel inlet, the tangential velocity was positive. However, in the wheel outlet, this compound became negative.

Figure 12 shows the distribution of the axial velocity. This compound was weak in the whole plane. However, between the blades 1 and 5, the axial velocity was 
not significant especially beside the hub.

The highest negative velocity was obtained in the upstream of the blade 1 .
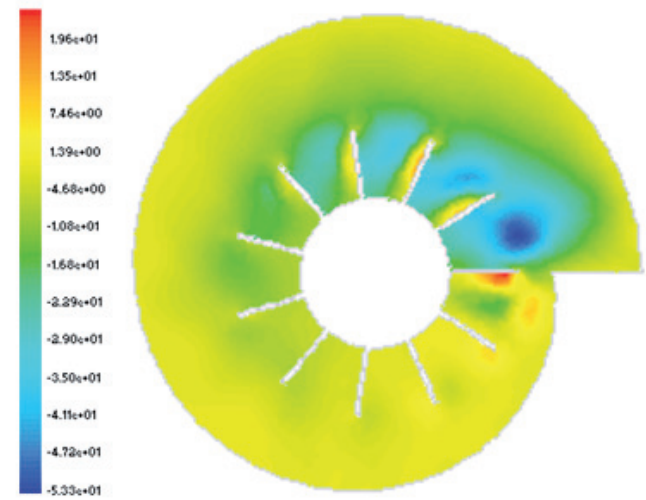

Fig. 10: Distribution of the radial velocity.
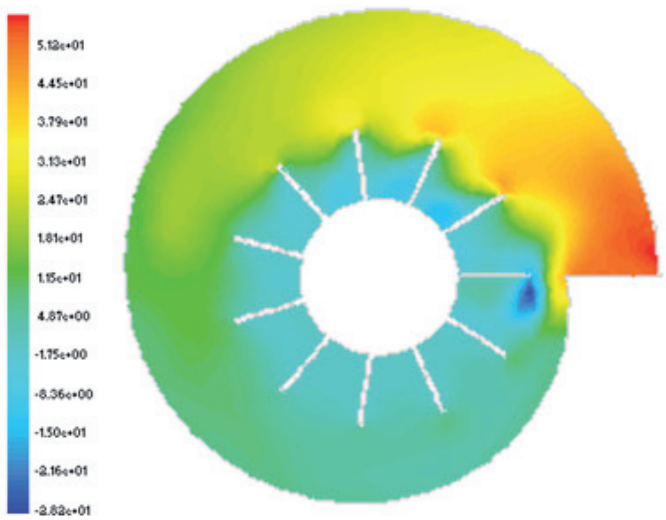

Fig. 11: Distribution of the tangential velocity.
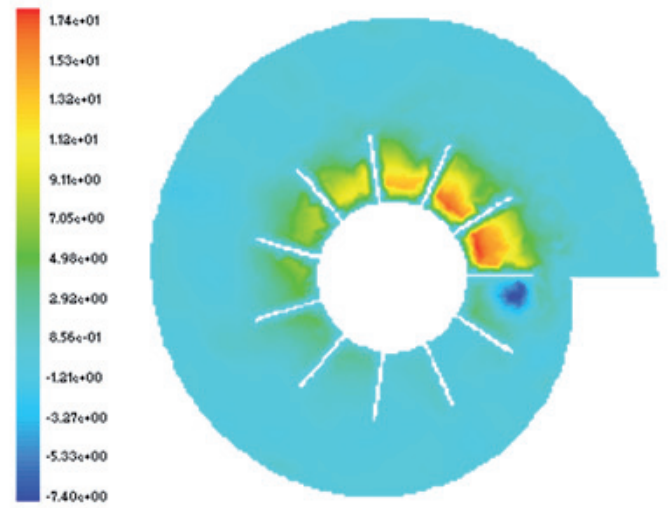

Fig. 12: Distribution of the axial velocity.
Figure 13 shows the distribution of the average velocity. On the walls of the volute and the blades, it was very weak. In addition, high gradients of the average velocity were located at the blades end. The maximum value was obtained in the end of the blade 2 . The flow was practically concentrated in the azimuth sector $0^{\circ}$ $180^{\circ}$. Out of this field, the flow was diminished and the velocity was very low.

Figure 14 shows the velocity vector plot of the flow. According to these results, there was formation of a recirculation zone in the downstream of the blades 2, 3 and 4 and in the air-gap between the blade 1 and the section volute inlet.
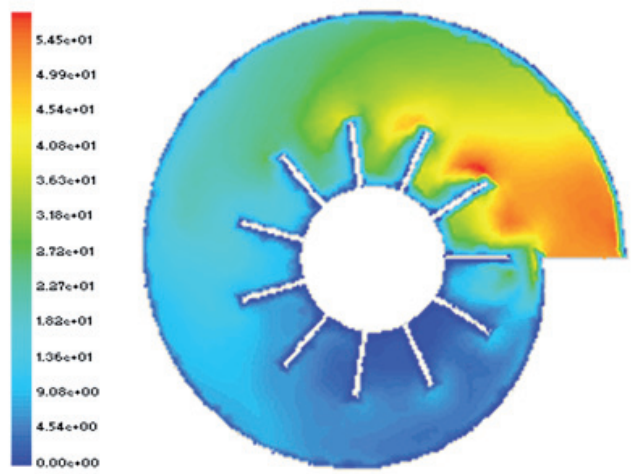

Fig. 13: Distribution of the average velocity.
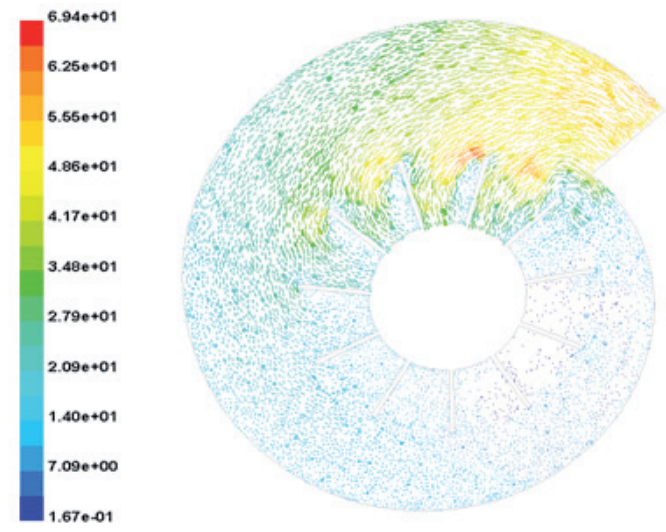

Fig. 14: Flows patterns.

Figure 15 shows the distribution of the turbulent kinetic energy $\mathrm{k}$. The highest values of $\mathrm{k}$ were located at the end of blade 1 presenting a stretch in the upstream. In the downstream of the blades 1,2 and 3, the values of $k$ were also enough significant and showed a wake effect. Out of this field, $\mathrm{k}$ remained low. 

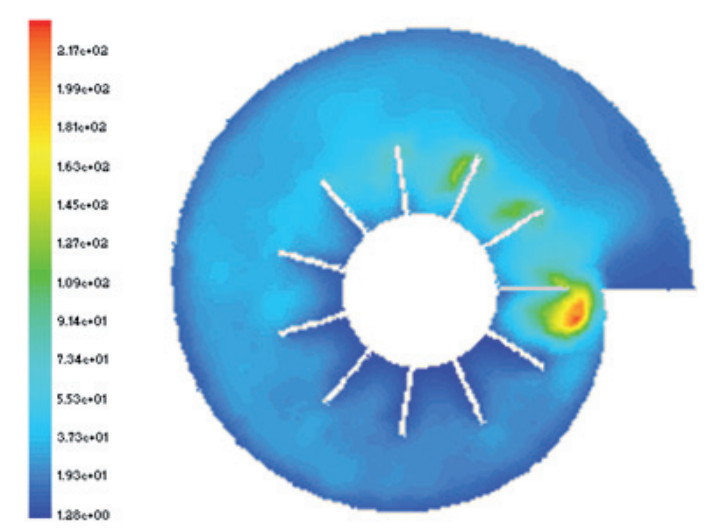

Fig. 15: Distribution of the turbulent kinetic energy $k$.

Figure 16 shows the distribution of the dissipation rate of the turbulent kinetic energy $\varepsilon$. According to these results, it has been noted that the areas of important values of $k$ were also the site of high values of $\varepsilon$. Out of this field, $\varepsilon$ was weak.

Figure 17 shows the distribution of the effective viscosity. Near the volute and blades walls, the values of the effective viscosity were weak. This figure shows also that the highest value of viscosity was where the turbulent kinetic energy is most important.
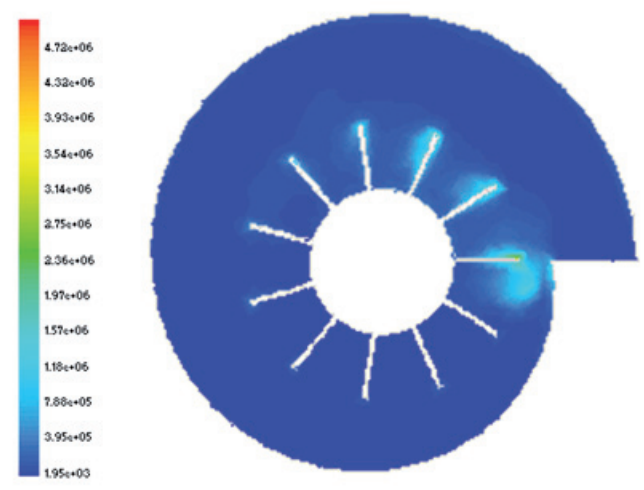

Fig. 16: Distribution of the dissipation rate of the turbulent kinetic energy $\varepsilon$.

\subsection{Flow characteristics in the turbine outlet}

In the turbine outlet, the evolution of the various characteristics of the flow is presented. Figures 18 and 19 show respectively the distribution of the axial and the average velocities in the turbine outlet. According to these results, it can be confirmed that the flow had a lateral and axial character and it was concentrated in an azimuth sector of $180^{\circ}$. These figures show also that the distribution of the average and the axial ve-

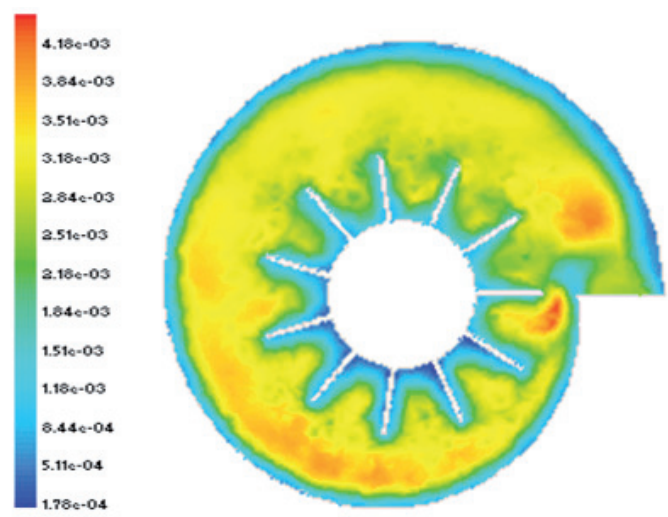

Fig. 17: Distribution of the effective viscosity ve.
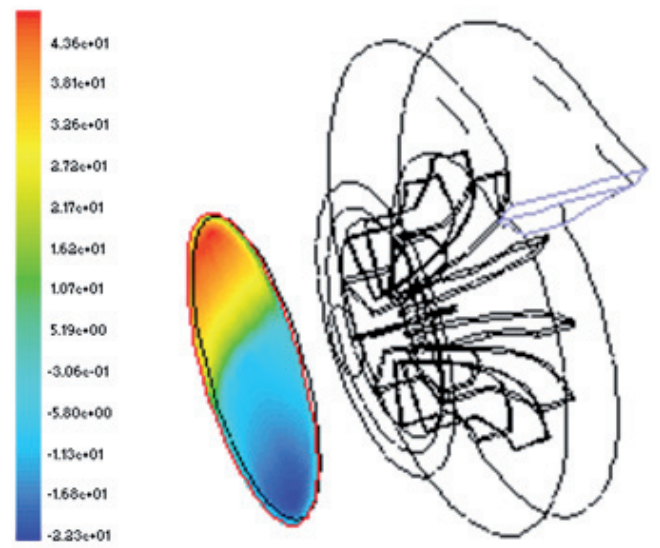

Fig. 18: Distribution of the axial velocity in the turbine outlet.
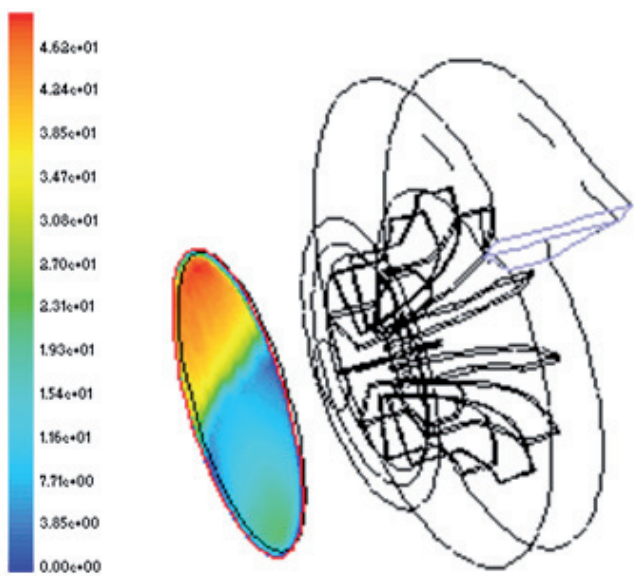

Fig. 19: Distribution of the velocity magnitude in the turbine outlet.

locities were not uniform in the turbine outlet. Figures 20, 21 and 22 show respectively the distribution of the turbulent kinetic energy $k$, the dissipation rate of the 
turbulent kinetic energy $\varepsilon$ and the effective viscosity ve in the turbine outlet. Figure 23 shows the evolution of the particle traces colored by average velocity from the volute inlet defined by $x=42$ and $y=z=0$ to the turbine outlet. This figure shows well the lateral character of the flow.

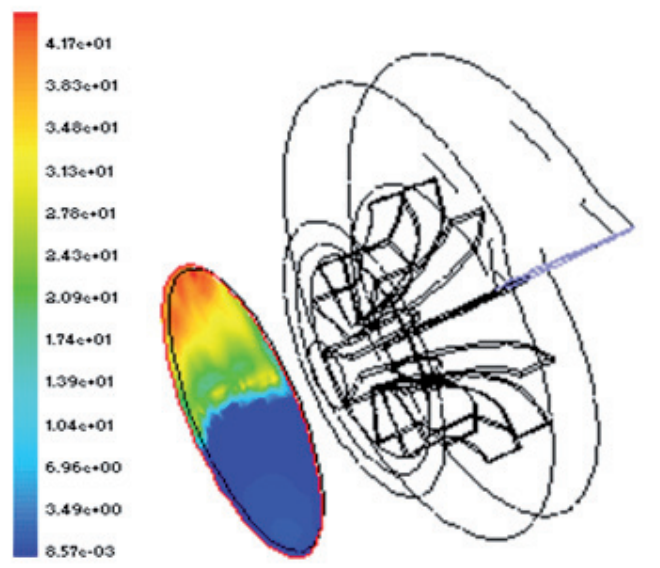

Fig. 20: Distribution of the turbulent kinetic energy $k$ in the turbine outlet.
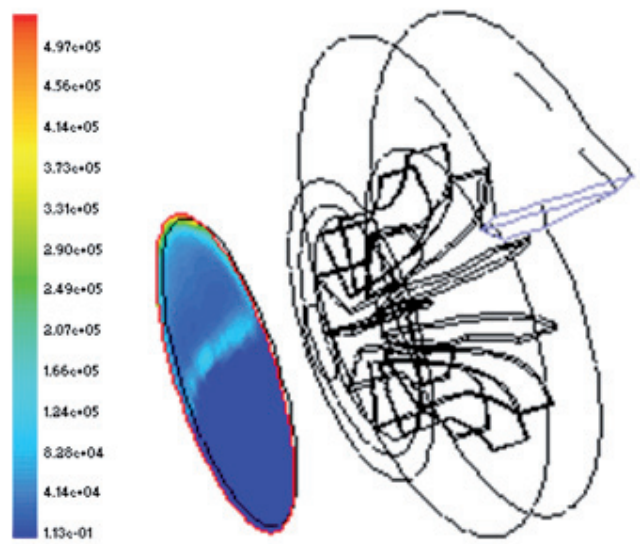

Fig. 21: Distribution of the dissipation rate of the turbulent kinetic energy $\varepsilon$ in the turbine outlet.

\section{Comparison with experimental results}

Figure 24 compares the detente rate $\mathrm{Pie} / \mathrm{Pis}$ as a function of the reduced mass flow $\operatorname{Dr}\left(\mathrm{kg} \cdot \mathrm{K}^{0.5} \cdot \mathrm{Pa}^{-1} \cdot \mathrm{s}^{-1}\right)$ obtained by the numerical study, to those found by Podevin application (Podevin, 1983). These points of operation of the turbine in the static state $(\mathrm{Va}=0)$ correspond to the inlet flows equal to 52,72 and 93 g.s s. $^{-1}$ The gap between the numerical and the experimental results was equal to $6 \%$. The obtained gap was rea-

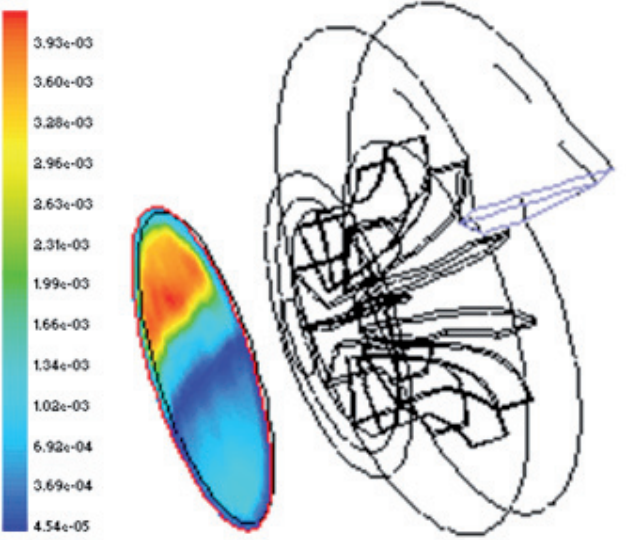

Fig. 22: Distribution of the effective viscosity ve in the turbine outlet.

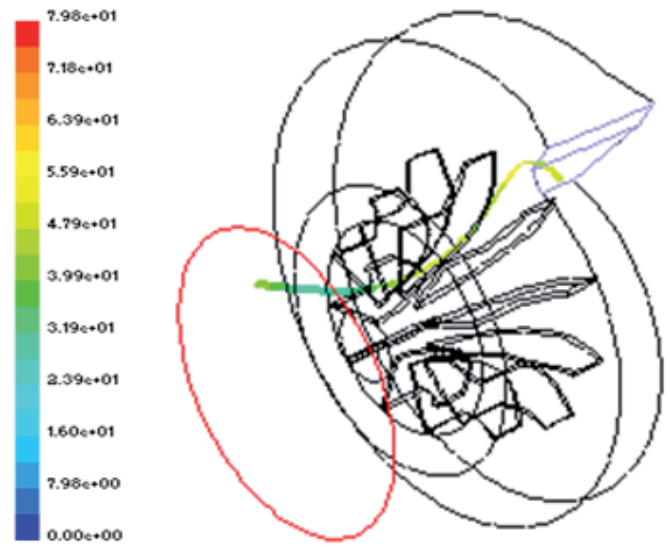

Fig. 23: Particle traces colored by velocity magnitude

- Numerical results

-Experimental results [8]

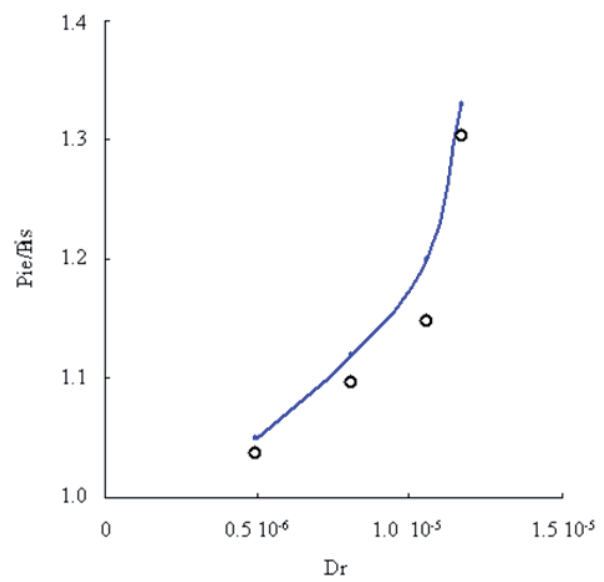

Fig. 24: Comparison of the numerical results with Podevin application (Podevin, 1983). 
sonable, and showed that our numerical study was an efficient tool to understand the flow throw the turbocharger turbine.

\section{Conclusion}

A numerical study of a turbocharger turbine in a static state was presented. The local features of the turbulent flow have been determined in the volute, the distributor, between blades of the wheel and the inlet and the outlet of the turbine. This modelling gives a fine knowledge of the hydrodynamic structures such as velocity, static pressure, turbulent kinetic energy, dissipation rate of the turbulent kinetic energy and effective viscosity. It also allows predicting, from the local results, the characteristic diagram operation of the turbocharger turbine. This knowledge can be exploited in future works to develop techniques to get an optimum design of the turbocharger turbines.

\section{References}

Driss Z., Mseddi M., Baccar M., Abid M. S., Caractérisation du fonctionnement d'une soupape de décharge de turbocompresseur de suralimentation. 4èmes Journées Scientifiques, Ecole de I'Aviation de Borj El-Amri, Tunisie, 2003, 2, pp.217-221.

Driss Z., Mseddi M., Baccar M., Abid M. S., Numerical and experimental study of a turbocharger's wastegate. International Conference on Advances in Mechanical Engineering (ICAME'04), Tunisia, 2004, T34, pp.1-6.

Fevre S., Application de la vélocimétrie Laser Doppler à des mesures de vitesses dans une petite turbine de suralimentation. Thèse de doctorat, Université Paris 6, 1990.

Frelin M., Podevin P., Modélisation des petites turbines centripètes. Revue Française de mécanique, 1992, 3, pp.257-265.

Friberg J., Mahieddinne M., Toussaint M. et Frelin M.: Prévision des caractéristiques des turbomachines Application à une pompe centrifuge, un compresseur centrifuge et une turbine centripète. Revue Française de Mécanique, 1988, 4, pp.55-66.

Katrasnik T., Improved model to determine turbine and compressor boundary conditions with the method of characteristics. International Journal of Mechanical Sciences, 2006, 48, pp.504-516.

Kesgin U., Study on the design of inlet and exhaust system of a stationary internal combustion engine. Energy Conversion and Management, 2005, 46, pp.2258-2287.

Kreuz-Ihli T., Filsinger D., Schulz A., Wittig S., Numerical and experimental study of unsteady flow field and vibration in radial inflow turbines. Journal of Turbomachinery, 2000, 122, pp.247-254.

Lee C.S., Choi N.J., Effect of air injection on the characteristics of transient response in a turbocharged diesel engine. Int. J. Therm. Sci., 2002, 41, pp.63-71.

Mazaud J.F., Influence of the supercharging system on the perfor- mance of a highly rated truck Diesel engine. S.I.A., 1990

Mseddi M., Baccar M., Kchaou H., Abid M. S., Modélisation des turbines radiales de suralimentation. Mécanique \& Industries, 2002, 3, pp.35-44.

Mseddi M., Contribution à l'étude des écoulements dans les turbomachines. Thèse de Doctorat d'état Es-Sciences Physiques, Faculté des Sciences de Sfax, Tunisia, 2004.

Mseddi M., Driss Z., Baccar M., Abid M. S., Etude d'une soupape de décharge de turbocompresseur de suralimentation. Mécanique \& Industries, 2005, 6, pp.539-551.

Mseddi M., Etude du couplage en régime quasi-stationnaire d'un turbocompresseur de suralimentation avec un moteur Diesel. Thèse de doctorat, Université Paris 6, 1990.

Okazaki Y., Development of variable area radial turbine for small turbochargers. A.S.M.E., 1992, 92.

Podevin P., Etude et essai d'une turbine radiale de turbocompresseur d'automobile, Mémoire d'Ingénieur, C.N.A.M. Paris, 1983.

Rakopoulos C.D., Giakoumis E.G., Availability analysis of a turbocharged diesel engine operating under transient load conditions, Energy, 2004, 29, pp.1085-1104.

Sarvi A., Fogelholm C.J., Zevenhoven R., Emissions from large-scale medium-speed diesel engines: 1. Influence of engine operation mode and turbocharger. Fuel Processing Technology, 2008, 89, pp.510-519.

Schorn N., Pischinger F., Schulte H., Computer simulation of turbocharged Diesel engines under transient conditions. Copyright Society of Automotive Engineers, 1987, pp.1-17.

Serrano J.R., Arnau F.J., Dolz V., Tiseira A., Cervelló C., A model of turbocharger radial turbines appropriate to be used in zero and one dimensional gas dynamics codes for internal combustion engines modelling. Energy Conversion and Management, 2008, 49, pp.3729-3745. 